\title{
A possible sets of diffuse bands originating at the same carrier
}

\author{
T. Weselak ${ }^{1}$, J. Fulara ${ }^{2}$, M. R. Schmidt ${ }^{3}$, and J. Krełowski ${ }^{1,4}$ \\ 1 Toruń Center for Astronomy, Nicolaus Copernicus University, Gagarina 11, 87-100 Toruń, Poland \\ e-mail: tomcat@astri.uni.torun.pl \\ ${ }^{2}$ Institute of Physics, Polish Academy of Sciences Al. Lotników 32/46, Pl-02-668 Warszawa, Poland \\ e-mail: fulara@ifpan.edu.pl \\ 3 Nicolaus Copernicus Astronomical Center, Rabiańska 8, Pl-87-100 Toruń, Poland \\ e-mail: schmidt@ncac.torun.pl \\ 4 Isaac Newton Institute of Chile, Polish Branch \\ e-mail: jacek@astri.uni.torun.pl
}

Received 6 February 2001 / Accepted 14 June 2001

\begin{abstract}
This paper discusses measurements of eight selected diffuse interstellar bands (DIBs): $\lambda \lambda 5793,5809$, $5819,5828,6196,6397,6614$ and 6660 performed in high resolution, high $S / N$ spectra of 41 reddened stars. Central depths, considered less error-prone than equivalent widths, are measured and mutual correlations between the selected DIBs are analyzed. Tight correlations between the DIBs: 5809, 6196, 6614 and 6660 may suggest their common origin despite their widths differing by a factor of up to 2 . The performed simulations prove that this fact does not preclude a common, molecular carrier of such features.
\end{abstract}

Key words. ISM: molecules - line: identification - methods: laboratory

\section{Introduction}

The first observational report of diffuse interstellar bands (DIBs) comes from early the twenties (Heger 1922) but the problem of identification of their carriers still remains unsolved. With the growing number of known DIBs, a tendency to gather them into "families" has appeared (Chlewicki et al. 1987; Krełowski \& Walker 1987). DIBs, being members of a family, preserve roughly constant intensity ratios from one line of sight to another. In some cases, the ratio of intensities of two DIBs varies drastically from one object to another, as has been shown by Krełowski \& Walker (1987) for the $\lambda 5780$ and $\lambda 5797$. Using the intensity ratio of the $\lambda 5780$ to $\lambda 5797$, Krełowski \& Walker (1987) have distinguished two types of interstellar clouds: $\sigma$-type, where the above ratio is high and $\zeta$-type, where it is low. The result proved that DIBs are not of the same origin and that environments of interstellar clouds are not the same: apparently physical parameters of individual clouds evidently may be different.

The spectra of $\zeta$-type clouds are particularly rich in DIBs. New surveys (Galazutdinov et al. 2000; O'Tuairisg 2000; Weselak et al. 2000) listed up to three hundred weak, unidentified spectral features that cover the visible and near infrared spectral region. Krełowski et al. (1997) have conjectured that weak diffuse bands are related to strong ones i.e. that spectra of single carriers

Send offprint requests to: T. Weselak, e-mail: tomcat@astri.uni.torun.pl consist of both strong and weak features. The problem of retrieving such spectra can be solved in two different ways. The first one is a normalization: the spectra of reddened stars that lie behind the $\zeta$ and/or $\sigma$ type clouds are normalized to the same central depth of one strong DIB (see Krełowski et al. 1997). Weak features, being of the same origin, should be also of the same depth in all selected and normalized spectra. The other way is to analyze correlations between central depths of DIBs; strengths of features of the same origin should correlate very tightly. We prefer using central depths for this analysis over equivalent widths as the latter cannot be measured precisely inside profiles of low $S / N$, which is always true in cases of weak DIBs. This method has recently been demonstrated by Motou et al. (1999).

The recent results showed that laboratory data nearly match several DIBs seen in the spectra of highly-reddened early-type stars. The origin of the two features at 9577 and $9632 \AA$, probably connected with the "buckminsterfullerene" $\mathrm{C}_{60}^{+}$, was discussed by Foing \& Ehrenfreund (1997) and Galazutdinov et al. (2000). The recent laboratory experiments showed that PAH ions could also explain the origin of some DIBs (Ehrenfreund et al. 1995). The comparison of laboratory spectra of PAH ions with observational data showed that only five species exhibit a positive correlation with DIBs (Salama et al. 1999). In the case of linear carbon chains, many coincidences between the wavelengths of their absorption features and the positions of diffuse bands have been found (Fulara et al. 1993). 
However, the spectra have been recorded in inert gas matrices. The spectra of $\mathrm{C}_{2 n}^{-}$and $\mathrm{C}_{2 n} \mathrm{H}$ have also been measured in the gas-phase and close matches of some absorption bands with the positions of DIBs have been found. A puzzling case is the electronic band of $\mathrm{NC}_{4} \mathrm{~N}^{+}$, which has a very similar shape to the $\lambda 5959$ DIB; however, the positions of these spectral features are mutually shifted by $0.9 \AA$ (Motylewski et al. 2000). The summary of laboratory measurements (in gas phase) concerning possible diffuse band carriers can be found in the review by Fulara \& Krełowski (2000).

An absorption spectrum of a DIB carrier, being a complicated carbon-bearing molecule, should contain one strong and several weak features, Fulara \& Krełowski (2000). The list of DIBs contains $\sim 30$ strong and $\sim 250$ weak features which qualitatively coincides with the above. The aim of this work is to connect strong and weak DIBs, possibly forming spectra of single carriers. The applied method is a search for DIBs that would preserve constant intensity ratios irrespective of observed objects possible sets of DIBs that originate from a single carrier each.

\section{The observational material}

Most of our observing material was gathered by one of us (JK) at the McDonald Observatory in Texas during 1993 using the Cassegrain echelle spectrograph fed from the 2.1-m telescope. The instrument consists of refractive collimator and camera optics, a 23.2 groves per mm echelle grating with a blaze angle of 65 degrees, a prism crossdisperser and a Reticon $400 \times 1200 \mathrm{CCD}$ with $27 \times 27 \mathrm{~m}$ pixels. The spectra have been recorded with the resolution equal to 64000 and a signal-to-noise ratio equal to 500 . Each spectrum covers the spectral range 5600-7000 $\AA$ in 26 orders. The instrument is described by McCarthy et al. (1993). The details of the method of data reduction of the spectra recorded with this instrument were described by Krełowski \& Sneden (1993).

The spectra of HD 186745 and additionally HD 183143, in the spectral range 4460-8800 $\AA$, were acquired with the aid of the new coude echelle spectrometer fed by the $2 \mathrm{~m}$ telescope of the observatory on the top of the peak Terskol (Northern Caucasia). For the data acquisition and reduction see Musaev (1993a) and Galazutdinov (1992). The data reduction of the echelle spectra was made using the DECH code (Galazutdinov 1992). This program allows location of the continuum, measurements of the line equivalent widths, line central depths, line positions and shifts, etc.

The stars, listed in Table 1 , are nearby, bright $\left(m_{\mathrm{v}}<\right.$ $7 \mathrm{mag}$ ), and medium-reddened. The observed colours have been taken from the SIMBAD database; the intrinsic colours taken from the paper of Papaj et al. (1993). In most of the spectra, atomic sodium lines $D_{1}$ and $D_{2}$ are sharp and thus these stars may be considered as obscured by a single, $\sigma$ or $\zeta$-type cloud. Our observational material involves only spectra containing the weak DIBs like $\lambda \lambda 5793$,
Table 1. The Stellar Data. The most of our observing material covers the spectral range 5600-7000 $\AA$; the stars whose spectra are in the range $4460-8800 \AA$ are marked with asterisks.

\begin{tabular}{|c|c|c|c|c|}
\hline $\mathrm{HD}$ & $\mathrm{Sp} / \mathrm{L}$ & $E(B-V)$ & $V$ & $\begin{array}{r}v \sin i \\
\mathrm{~km} \mathrm{~s}^{-1}\end{array}$ \\
\hline 2905 & B1I & 0.33 & 4.16 & 62 \\
\hline 8065 & $\mathrm{~A} 0 \mathrm{I}$ & 0.39 & 6.46 & \\
\hline 12953 & A1I & 0.62 & 6.28 & 30 \\
\hline 13267 & B5I & 0.41 & 6.36 & 53 \\
\hline 14489 & A2I & 0.40 & 5.17 & 25 \\
\hline 20041 & $\mathrm{~A} 0 \mathrm{I}$ & 0.73 & 5.79 & \\
\hline 21291 & B9I & 0.42 & 4.21 & 29 \\
\hline 21389 & A0Iae & 0.56 & 4.54 & 6 \\
\hline 24534 & O9.5pe & 0.56 & 6.10 & 150 \\
\hline 41117 & B2Ia & 0.44 & 4.63 & 36 \\
\hline 42087 & $\mathrm{~B} 2.5 \mathrm{I}$ & 0.35 & 5.75 & 37 \\
\hline 43384 & B3I & 0.58 & 6.25 & 51 \\
\hline 47129 & $\mathrm{O} 8 \mathrm{~V}$ & 0.33 & 6.06 & 80 \\
\hline 54662 & O7III & 0.27 & 6.21 & 91 \\
\hline 144217 & B0.5V & 0.17 & 2.62 & 130 \\
\hline 144470 & B1V & 0.19 & 3.96 & 142 \\
\hline 145502 & B3V & 0.25 & 4.01 & 199 \\
\hline 147165 & $\mathrm{~B} 2 \mathrm{III}+\mathrm{O} 9.5 \mathrm{~V}$ & 0.36 & 2.89 & 53 \\
\hline 147933 & B2IV & 0.44 & 5.02 & 303 \\
\hline 148184 & B2IV & 0.48 & 4.42 & 134 \\
\hline 149757 & $09.5 \mathrm{~V}$ & 0.29 & 2.56 & 379 \\
\hline 154445 & $\mathrm{~B} 1 \mathrm{~V}$ & 0.39 & 5.64 & 174 \\
\hline 166937 & B2IV-V & 0.30 & 5.82 & 120 \\
\hline 179406 & B3V & 0.31 & 5.34 & 187 \\
\hline$\star 183143$ & B7Iae & 1.28 & 6.86 & 59 \\
\hline 184915 & B0.5III & 0.22 & 4.95 & 259 \\
\hline$\star 186745$ & B8Ia & 0.95 & 7.07 & \\
\hline 198478 & B3Iae & 0.54 & 4.84 & 35 \\
\hline 199579 & O6Ve & 0.34 & 5.96 & 170 \\
\hline 203064 & O8e & 0.28 & 5.00 & 328 \\
\hline 206165 & $\mathrm{~B} 2 \mathrm{Ib}$ & 0.46 & 4.73 & 36 \\
\hline 206267 & O6f & 0.50 & 5.62 & 154 \\
\hline 207198 & O9II & 0.56 & 5.95 & 76 \\
\hline 207260 & A2Ia & 0.50 & 4.29 & 33 \\
\hline 208501 & B8Ib & 0.75 & 5.80 & 53 \\
\hline 209481 & O9V & 0.34 & 5.56 & 130 \\
\hline 209975 & O9I & 0.33 & 5.11 & 33 \\
\hline 210839 & O6If & 0.46 & 5.04 & 285 \\
\hline 218376 & B0.5IV & 0.21 & 4.85 & 50 \\
\hline 223128 & B2IV & 0.16 & 5.95 & \\
\hline 224572 & $\mathrm{~B} 1 \mathrm{~V}$ & 0.16 & 4.88 & 189 \\
\hline
\end{tabular}

5809, 5819, 5828 and 6397. A few highly reddened stars are also present, to show how the correlation plots extrapolate to high colour excesses. The spectra of highly reddened HD 183143 and 186745 include also the region 4460-8800 $\AA$ (only a few DIBs have been listed out of this range) to test whether any additional bands 
connected with those, well correlated in the spectral region of McDonald spectra (5600-7000 $)$, can be found.

\subsection{Searching for DIBs possibly originating at the same carrier}

The survey of Weselak et al. (2000) was based on two averaged spectra involving many $\sigma$ and $\zeta$-type clouds. These spectra have been normalized to the same depth of the $6614 \AA$ band. Then we have examined them, searching for diffuse bands (strong and weak) being of nearly the same central depths in both averaged spectra. The selected DIBs that fulfil this criterion are following: $\lambda \lambda 5793$, 5809, 5819, 5828, 6397, 6196, 6614 and 6660 .

In the spectra of heavily reddened stars HD 183143 and 186745 , which resemble the "sigma" and the "zeta" type objects respectively, the $\lambda 6614$ band is of nearly the same central depth, which allows a direct comparison of the spectra without any normalization. The spectra of HD 183143 and HD 186745 (acquired at the Terskol Observatory) have been used to search for other DIBs that would lie outside of the range covered by the McDonald instrument and would be of the same intensity in the spectra of these stars (which may suggest they originate in the same carrier). Two additional bands at 5404.5 and $7257.5 \AA$ selected in this way were found in the range 4460-8800 $\AA$. It is, however, necessary to analyze mutual correlations between the central depths of the DIBs: $\lambda \lambda$ 5404.5, 5793, 5809, 5819, 5828, 6397, 6196, 6614, 6660 and 7257.5 to prove that they originate in the same carrier.

\subsection{The measurements. Central depths or equivalent widths}

In most of cases diffuse bands are very weak and thus very shallow, which cause difficulties when detecting them in spectra of low signal-to-noise ratio. To analyze mutual correlations between DIBs, we present their central depth measurements in \% of the continuum in the case of $\lambda \lambda 5793,5809,5819,5828,6196,6397,6614,6660$ (Table 2). Also, the equivalent width measurements acquired for $\lambda \lambda 5809,6614$ are given in Table 2; the following codes are used: $\mathrm{n} / \mathrm{s}$ - structure not seen; $\mathrm{bl}$ - structure blended with a stellar line and $\mathrm{n} / \mathrm{a}$ - spectrum not available. The errors of central depth measurements are caused by uncertain placement of the continuum level. They also depend on the quality of each echelle spectrum. In the case of equivalent width measurements, each DIB profile was fitted with a Gaussian curve. As we present in Figs. 1 and 2, which show correlation plots between $\lambda \lambda 5809$ and 6614, their central depths correlate much better than $E W$ 's do. This demonstrates that central depth measurements can be measured more precisely than equivalent widths. Errors originating in the continuum displacement for equivalent width measurements have higher values than those in the case of central depths.

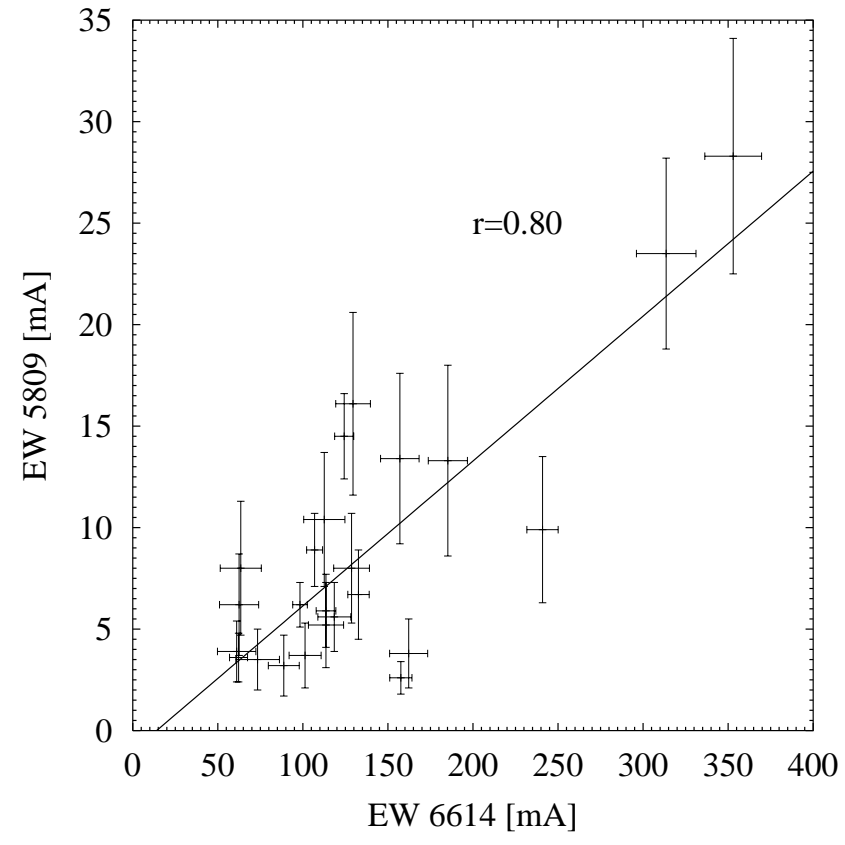

Fig. 1. The correlation plot between equivalent widths of $\lambda \lambda 6614 / 5809$.

\subsection{Additional effects seen in the spectra of target stars}

Each measurement of a spectral feature may suffer errors resulting from several possible effects: blending of diffuse bands with stellar and/or interstellar features and some unknown instrumental effects. In the case of DIBs situated in the red and infrared spectra, detected with back illuminated CCDs, the effect of fringing is substantial. As a result, the correlation of any two features, originating in the same carrier, may be relatively weak. To achieve a reasonably good mutual correlation between central depths or equivalent widths of DIBs (especially weak ones), the spectra of reddened stars ought to be of high quality. The central depths of most of the observed features are of the order of one percent of continuum and thus the $S / N$ ratio inside DIB profiles is always low.

The data reduction is also important. It is difficult to guarantee that the divisor spectrum (used to remove telluric lines from the considered spectra) lacks any weak stellar or interstellar features that would contaminate the profiles of diffuse bands. One of the possible effects, easily seen in the spectra of stars with small rotational velocities, is the effect of blending of the diffuse band profiles with stellar lines. In Fig. 3 we present the region of the weak diffuse band $\lambda 6397$, seen in the spectra of four stars from our sample. The central depths of $\lambda 6397$, as observed in the two sets of spectra, seem to be similar, but the profiles observed in the case of HD 206165 and 209975 are of different widths. The synthetic spectrum (see references in Weselak et al. 2000) for B2I-type star with the rotational velocity of $80 \mathrm{~km} \mathrm{~s}^{-1}$ (at the top of this figure) shows the stellar line of S II at $6397.395 \AA$. The $\lambda 6397$ DIB seen in the spectrum of HD 206165, the object of small rotational 
Table 2. The measurements of central depths in $\%$ of the continuum level and equivalent widths in $\mathrm{m} \AA$. In the table the following codes are used: $\mathrm{n} / \mathrm{s}$ - structure not seen; bl - structure blended with a stellar line and $\mathrm{n} / \mathrm{a}$ - spectrum not available.

\begin{tabular}{|c|c|c|c|c|c|c|c|c|c|c|}
\hline $\mathrm{HD}$ & $\lambda 5793$ & $\lambda 5809$ & $\lambda 5819$ & $\lambda 5828$ & $\lambda 6196$ & $\lambda 6397$ & $\lambda 6614$ & $\lambda 6660$ & $\begin{array}{c}\lambda 5809 \\
\mathrm{~m} \AA\end{array}$ & $\begin{array}{c}\lambda 6614 \\
\mathrm{~m} \AA\end{array}$ \\
\hline 2905 & $0.5(0.1)$ & $1.0(0.1)$ & $0.7(0.2)$ & $\mathrm{n} / \mathrm{s}$ & $7.1(0.2)$ & $1.2(0.1)$ & $12.4(0.4)$ & $4.1(0.2)$ & $16.1(4.5)$ & $129.5(10.2)$ \\
\hline 8065 & $0.5(0.1)$ & $\mathrm{n} / \mathrm{s}$ & $\mathrm{n} / \mathrm{s}$ & $\mathrm{n} / \mathrm{s}$ & $4.5(0.2)$ & $1.2(0.1)$ & $8.7(0.2)$ & $3.2(0.1)$ & $\mathrm{n} / \mathrm{s}$ & $74.4(9)$ \\
\hline 12953 & $0.8(0.2)$ & $0.7(0.1)$ & $1.3(0.2)$ & $\mathrm{n} / \mathrm{s}$ & $4.3(0.4)$ & $2.1(0.2)$ & $10.7(0.3)$ & $\mathrm{n} / \mathrm{a}$ & $3.2(1.5)$ & $88.8(9.1)$ \\
\hline 13267 & $1.1(0.1)$ & $0.8(0.1)$ & $1.9(0.3)$ & $\mathrm{n} / \mathrm{s}$ & $5.0(0.2)$ & $2.6(0.2)$ & $10.1(0.2)$ & $\mathrm{n} / \mathrm{a}$ & $5.6(1.7)$ & $118.5(9.7)$ \\
\hline 14489 & $0.6(0.1)$ & $1.0(0.1)$ & $0.8(0.2)$ & $\mathrm{n} / \mathrm{s}$ & $4.9(0.2)$ & $0.9(0.2)$ & $9.9(0.2)$ & $4.1(0.2)$ & $5.2(2.1)$ & $113.6(10.3)$ \\
\hline 20041 & $1.0(0.2)$ & $1.7(0.1)$ & $0.7(0.2)$ & $0.9(0.2)$ & $8.4(0.5)$ & $2.5(0.3)$ & $22.6(0.3)$ & $7.8(0.3)$ & $9.9(3.6)$ & $240.9(9.2)$ \\
\hline 21291 & $0.7(0.1)$ & $1.0(0.1)$ & $1.2(0.2)$ & $0.5(0.2)$ & $4.4(0.3)$ & $1.0(0.2)$ & $9.6(0.2)$ & $4.0(0.2)$ & $3.7(1.6)$ & $101.3(9.4)$ \\
\hline 21389 & $0.8(0.2)$ & $1.2(0.1)$ & $0.7(0.2)$ & $0.9(0.2)$ & $6.3(0.3)$ & $1.6(0.3)$ & $15.4(0.3)$ & $5.2(0.3)$ & $3.8(1.7)$ & $162.2(11.2)$ \\
\hline 24534 & $0.8(0.1)$ & $\mathrm{n} / \mathrm{s}$ & $\mathrm{n} / \mathrm{s}$ & $\mathrm{n} / \mathrm{s}$ & $2.9(0.2)$ & $1.0(0.2)$ & $5.1(0.3)$ & $\mathrm{n} / \mathrm{a}$ & $\mathrm{n} / \mathrm{s}$ & $72.8(10.5)$ \\
\hline 41117 & $1.1(0.2)$ & $1.4(0.1)$ & $\mathrm{n} / \mathrm{s}$ & $\mathrm{n} / \mathrm{s}$ & $8.2(0.1)$ & $1.9(0.3)$ & $16.2(0.4)$ & $4.9(0.3)$ & $13.4(4.2)$ & $157.0(11.3)$ \\
\hline 42087 & $1.8(0.2)$ & $0.8(0.1)$ & $1.6(0.3)$ & $1.7(0.3)$ & $6.7(0.3)$ & $1.9(0.2)$ & $9.9(0.4)$ & $2.8(0.3)$ & $8.0(2.7)$ & $128.6(10.5)$ \\
\hline 43384 & $2.1(0.3)$ & $1.3(0.1)$ & bl & $0.8(0.5)$ & $11.4(0.3)$ & $2.0(0.3)$ & $18.4(0.4)$ & $5.7(0.3)$ & $13.3(4.7)$ & $185.2(11.5)$ \\
\hline 47129 & $\mathrm{n} / \mathrm{s}$ & $\mathrm{n} / \mathrm{s}$ & $\mathrm{n} / \mathrm{s}$ & $0.6(0.2)$ & $4.4(0.2)$ & $\mathrm{n} / \mathrm{s}$ & $8.5(0.5)$ & $1.9(0.3)$ & $\mathrm{n} / \mathrm{s}$ & 76.9 (11.1) \\
\hline 54662 & $\mathrm{n} / \mathrm{s}$ & $\mathrm{bl}$ & $0.8(0.2)$ & $\mathrm{n} / \mathrm{s}$ & $4.8(0.3)$ & $1.4(0.2)$ & $8.3(0.4)$ & $3.4(0.5)$ & $\mathrm{bl}$ & $98.3(14.2)$ \\
\hline 144217 & $\mathrm{n} / \mathrm{s}$ & $\mathrm{n} / \mathrm{s}$ & $0.4(0.2)$ & $0.4(0.1)$ & $3.1(0.2)$ & $\mathrm{n} / \mathrm{s}$ & $4.7(0.3)$ & $\mathrm{n} / \mathrm{s}$ & $\mathrm{n} / \mathrm{s}$ & $53.8(5.6)$ \\
\hline 144470 & $0.4(0.1)$ & $0.7(0.1)$ & $0.4(0.2)$ & $0.5(0.2)$ & $\mathrm{n} / \mathrm{a}$ & $\mathrm{n} / \mathrm{s}$ & $5.5(0.2)$ & $1.5(0.2)$ & $8.0(3.3)$ & $63.5(12.1)$ \\
\hline 145502 & $0.4(0.1)$ & $0.5(0.1)$ & $\mathrm{n} / \mathrm{s}$ & $\mathrm{n} / \mathrm{s}$ & $3.2(0.2)$ & $\mathrm{n} / \mathrm{s}$ & $5.1(0.5)$ & $1.6(0.2)$ & $3.9(1.5)$ & $61.1(11.3)$ \\
\hline 147165 & $\mathrm{n} / \mathrm{s}$ & $0.7(0.1)$ & $\mathrm{n} / \mathrm{s}$ & $0.5(0.2)$ & $4.1(0.3)$ & $\mathrm{n} / \mathrm{s}$ & $5.3(0.4)$ & $1.4(0.3)$ & $6.2(2.5)$ & $62.5(11.5)$ \\
\hline 147933 & $0.9(0.1)$ & $0.8(0.1)$ & $1.2(0.2)$ & $1.5(0.2)$ & $3.3(0.3)$ & $1.2(0.3)$ & $5.9(0.3)$ & $1.7(0.2)$ & $3.5(1.5)$ & $73.4(12.8)$ \\
\hline 148184 & $0.9(0.1)$ & $0.4(0.1)$ & $0.5(0.2)$ & $0.8(0.2)$ & $2.6(0.2)$ & $1.0(0.2)$ & $4.4(0.2)$ & $1.3(0.3)$ & $\mathrm{n} / \mathrm{s}$ & $41.6(9.8)$ \\
\hline 149757 & $0.4(0.1)$ & bl & bl & $0.7(0.2)$ & $2.7(0.2)$ & $\mathrm{n} / \mathrm{s}$ & $4.6(0.3)$ & $1.0(0.3)$ & $\mathrm{bl}$ & 42.9 (12.0) \\
\hline 154445 & $\mathrm{n} / \mathrm{s}$ & $0.9(0.1)$ & $0.6(0.2)$ & $0.8(0.2)$ & $6.0(0.2)$ & $1.5(0.1)$ & $11.2(0.5)$ & $3.6(0.3)$ & $10.4(3.3)$ & $112.6(12.1)$ \\
\hline 166937 & $0.6(0.1)$ & $0.8(0.1)$ & $1.3(0.2)$ & $1.0(0.2)$ & $5.8(0.2)$ & $1.9(0.3)$ & $9.1(0.7)$ & $2.6(0.3)$ & $8.9(1.8)$ & $106.9(4.7)$ \\
\hline 179406 & $1.0(0.1)$ & $1.0(0.1)$ & $0.8(0.2)$ & $1.1(0.2)$ & $4.8(0.2)$ & $1.6(0.2)$ & $9.6(0.3)$ & $4.3(0.3)$ & $6.2(1.1)$ & $98.3(4.3)$ \\
\hline$\star 183143$ & $2.1(0.3)$ & $2.3(0.1)$ & $1.3(0.2)$ & $1.7(0.2)$ & $14.5(0.4)$ & $3.2(0.3)$ & $31(0.7)$ & $12.2(0.5)$ & $28.3(5.8)$ & $353.0(16.7)$ \\
\hline 184915 & $\mathrm{n} / \mathrm{s}$ & $0.6(0.1)$ & $\mathrm{n} / \mathrm{s}$ & $\mathrm{n} / \mathrm{s}$ & $4.4(0.3)$ & $0.9(0.2)$ & $6.9(0.2)$ & $3.0(0.2)$ & $\mathrm{n} / \mathrm{s}$ & $75.4(15.5)$ \\
\hline$\star 186745$ & $3.5(0.2)$ & $2.7(0.2)$ & $3.3(0.3)$ & $3.2(0.4)$ & $14.8(0.4)$ & $4.6(0.2)$ & $29.8(0.4)$ & $13.0(0.3)$ & $23.5(4.7)$ & $313.6(17.5)$ \\
\hline 198478 & $0.7(0.2)$ & $1.1(0.2)$ & $1.6(0.2)$ & $0.9(0.2)$ & $7.2(0.3)$ & $1.7(0.2)$ & $13.2(0.5)$ & $3.7(0.2)$ & $6.7(2.2)$ & $132.7(6.3)$ \\
\hline 199579 & $\mathrm{n} / \mathrm{s}$ & $\mathrm{bl}$ & $1.3(0.4)$ & $1.3(0.4)$ & $3.5(0.4)$ & $\mathrm{n} / \mathrm{s}$ & $4.8(0.8)$ & $\mathrm{n} / \mathrm{a}$ & $\mathrm{bl}$ & $62.2(6.4)$ \\
\hline 203064 & $\mathrm{n} / \mathrm{s}$ & $\mathrm{bl}$ & $0.8(0.3)$ & $0.6(0.2)$ & $4.0(0.4)$ & $1.4(0.1)$ & $6.7(0.4)$ & $1.6(0.2)$ & $\mathrm{bl}$ & $71.4(5.9)$ \\
\hline 206165 & $0.8(0.1)$ & $0.8(0.1)$ & $1.3(0.3)$ & $0.9(0.3)$ & $5.8(0.3)$ & $1.5(0.1)$ & $10.3(0.4)$ & $3.4(0.2)$ & $5.9(1.8)$ & $113.6(5.8)$ \\
\hline 206267 & $0.9(0.2)$ & $\mathrm{bl}$ & $0.9(0.2)$ & $1.3(0.3)$ & $6.2(0.4)$ & $1.5(0.1)$ & $11.6(0.3)$ & $3.5(0.4)$ & bl & $105.9(5.2)$ \\
\hline 207198 & $1.4(0.2)$ & bl & $1.4(0.2)$ & $1.8(0.3)$ & $6.9(0.4)$ & $2.0(0.2)$ & $13.0(0.3)$ & $3.8(0.4)$ & bl & $121.4(5.7)$ \\
\hline 207260 & $0.9(0.2)$ & $0.8(0.1)$ & $1.0(0.3)$ & $0.6(0.2)$ & $7.3(0.4)$ & $1.7(0.1)$ & $13.7(0.4)$ & $4.9(0.2)$ & $2.6(0.8)$ & $157.6(6.5)$ \\
\hline 208501 & $0.8(0.2)$ & $1.2(0.1)$ & $2.1(0.2)$ & $1.3(0.2)$ & $6.5(0.3)$ & $2.7(0.4)$ & $12.0(0.3)$ & $5.1(0.3)$ & $14.5(2.1)$ & $124.3(5.6)$ \\
\hline 209481 & $0.8(0.2)$ & bl & $\mathrm{n} / \mathrm{s}$ & $0.8(0.1)$ & $5.3(0.3)$ & $1.5(0.2)$ & $9.7(0.7)$ & $3.0(0.5)$ & bl & $96.5(5.4)$ \\
\hline 209975 & $1.0(0.2)$ & $\mathrm{bl}$ & $0.8(0.2)$ & $0.9(0.2)$ & $6.4(0.5)$ & $1.5(0.1)$ & $11.3(0.5)$ & $3.4(0.1)$ & $\mathrm{bl}$ & $109.6(5.8)$ \\
\hline 210839 & $0.7(0.1)$ & $\mathrm{bl}$ & $0.8(0.2)$ & $1.0(0.2)$ & $7.9(0.2)$ & $1.7(0.1)$ & $15.1(0.5)$ & $4.6(0.4)$ & bl & $138.3(5.7)$ \\
\hline 218376 & $0.6(0.1)$ & $0.7(0.1)$ & $0.7(0.2)$ & $\mathrm{n} / \mathrm{s}$ & $3.8(0.2)$ & $0.8(0.1)$ & $6.9(0.4)$ & $1.8(0.2)$ & $3.6(1.2)$ & $62.2(5.3)$ \\
\hline 223128 & $0.7(0.1)$ & $\mathrm{n} / \mathrm{s}$ & $\mathrm{n} / \mathrm{s}$ & $\mathrm{n} / \mathrm{s}$ & $4.1(0.2)$ & $1.5(0.2)$ & $7.1(0.3)$ & $2.7(0.2)$ & bl & $53.7(3.5)$ \\
\hline 224572 & $\mathrm{n} / \mathrm{s}$ & $\mathrm{n} / \mathrm{s}$ & $\mathrm{n} / \mathrm{s}$ & $\mathrm{n} / \mathrm{s}$ & $2.6(0.2)$ & $\mathrm{n} / \mathrm{s}$ & $4.2(0.4)$ & $1.5(0.2)$ & $\mathrm{bl}$ & $36.3(4.3)$ \\
\hline
\end{tabular}

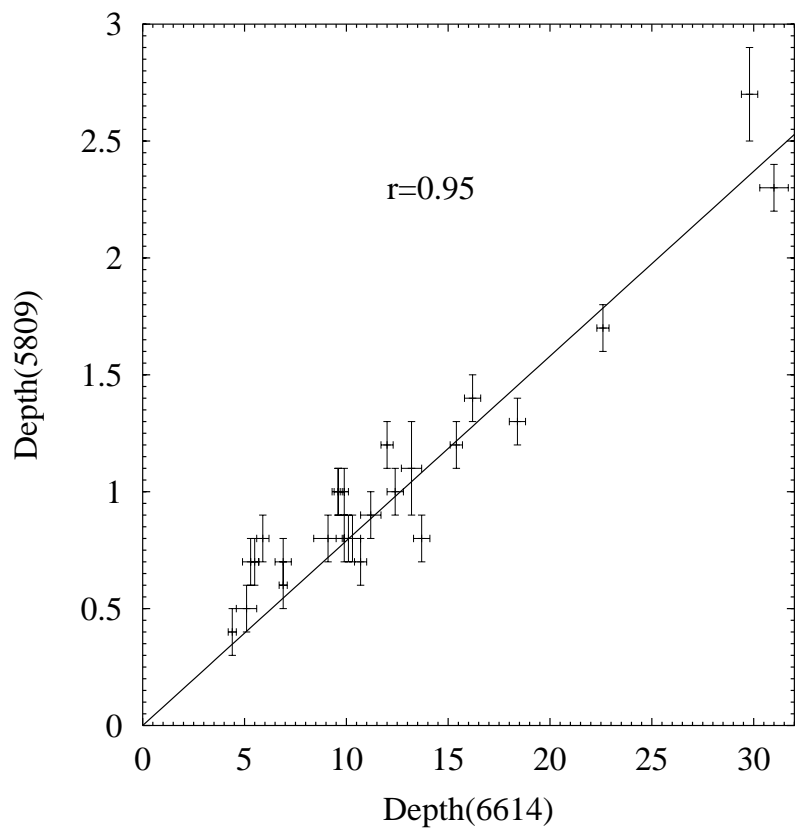

Fig. 2. The correlation plot between central depths of $\lambda \lambda 6614 / 5809$.

velocity, is blended with this stellar feature. In the spectra of two heavily reddened stars, HD 183143 and 186745, the diffuse band at $6397 \AA$ is seen without any difficulty and is possibly not blended with a stellar line. In the case of two spectra, seen at the top of this figure, the stars HD 206165 and HD 209975 are of nearly the same $E(B-V)$ ratio as that of HD 183143 and 186745. The central depths of $\lambda 6397$, as seen in the pairs of the spectra, have also nearly the same value.

Since the publication of Herbig \& Soderblom (1982) it is known that the Doppler splitting, seen in the sharp profiles of interstellar atomic lines, also affects the profiles of diffuse bands. DIBs observed in the spectra of heavily reddened and distant stars are composed of multiple components. Each component originates in an individual cloud of possibly different physical conditions and spectrum. In the case of broader DIBs the Doppler splitting cannot be seen directly, but the resultant profiles are much broader than in a case of a single cloud (Westerlund \& Krełowski 1988).

In Fig. 4 we present the spectral region containing interstellar sodium lines $D_{1}$ and $D_{2}$ as seen in the spectra of four stars presented in Fig. 3 and additionally in the spectrum of HD 147165. The sodium lines show two components in the case of the heavily reddened star HD 183143; in the case of HD 186745, 206165 and 209975 the profiles are much broader than those of interstellar sodium lines 


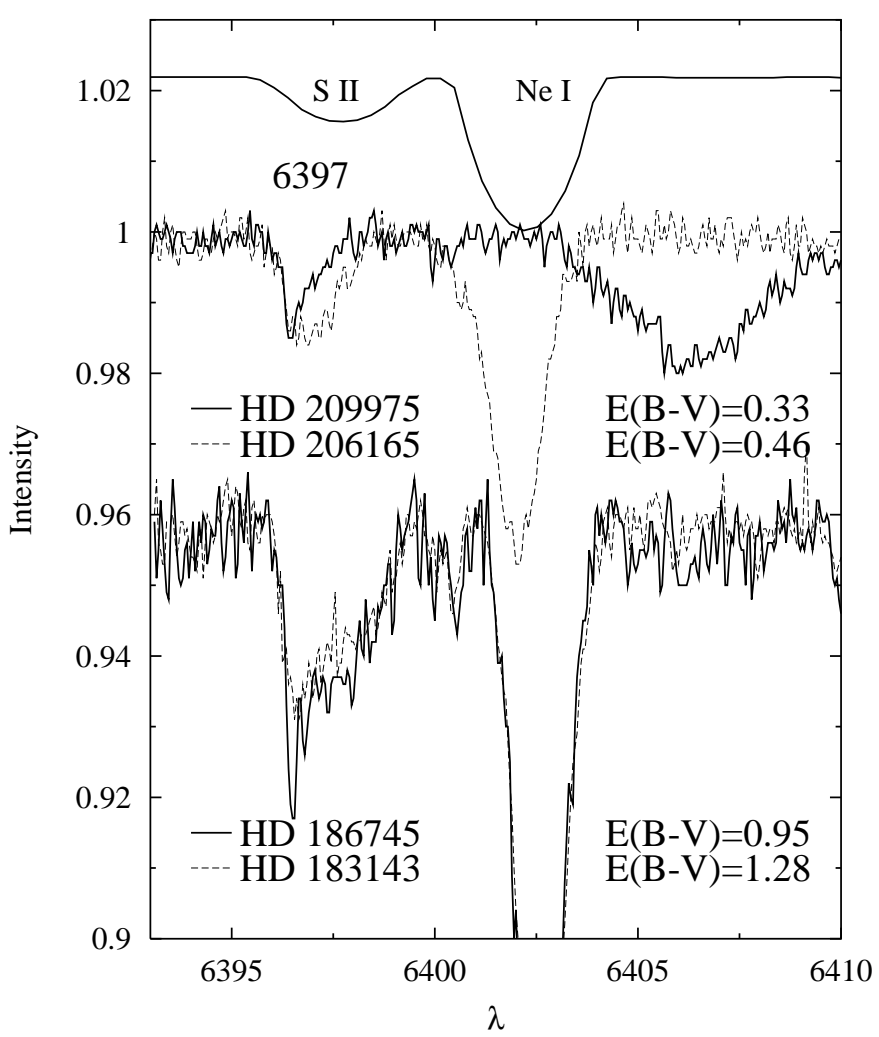

Fig. 3. The region of $\lambda 6397$ in the two pairs of spectra. Note the Ne I line at $6402.246 \AA$ seen in the spectra of B-type stars and the stellar line of S II at $6397.355 \AA$ Alended with the $\lambda 6397$ profile of HD 206165. The $E(B-V)$ ratios in the case of the two pairs of spectra are nearly the same and the central depths of $\lambda 6397$ in the pairs of spectra are also similar.

observed in a single cloud (see the profiles of $\mathrm{Na}$ I lines in the spectrum of HD 147165 from the Sco OB2 association at the bottom of this figure). The two components, seen in the profiles of sodium lines in the spectrum of HD 183143, are also seen in the $\lambda 6614$ profile in Fig. 5.

It is evident (Fig. 5) that in the spectra of $\sigma$-type HD 183143 and $\zeta$-type HD 186745 (Fig. 6) the central depths of $\lambda 6614$ are quite similar despite being formed in different physical conditions. Any other features, being of similar strengths in these two spectra, are very likely to originate in the same carrier.

\section{Results}

\subsection{Correlation analysis}

The procedure of obtaining correlation coefficients is fully described by Motou et al. (1999). On the basis of this we have obtained tight correlations (i.e. with $r>0.95)$ between the DIB pairs $\lambda \lambda 6614 / 6196$, $6660 / 5809$ and $\lambda \lambda 6614 / 6660$. The other important cases are: $\lambda \lambda 6614 / 5809(r=0.95), \lambda \lambda 6196 / 6660(r=0.92)$ and $\lambda \lambda 6196 / 5809(r=0.90)$. In Table 3 we present the values of correlation coefficients in the case of each pair of DIBs. Generally, the correlation coefficients of relations between stronger diffuse bands are of higher values than

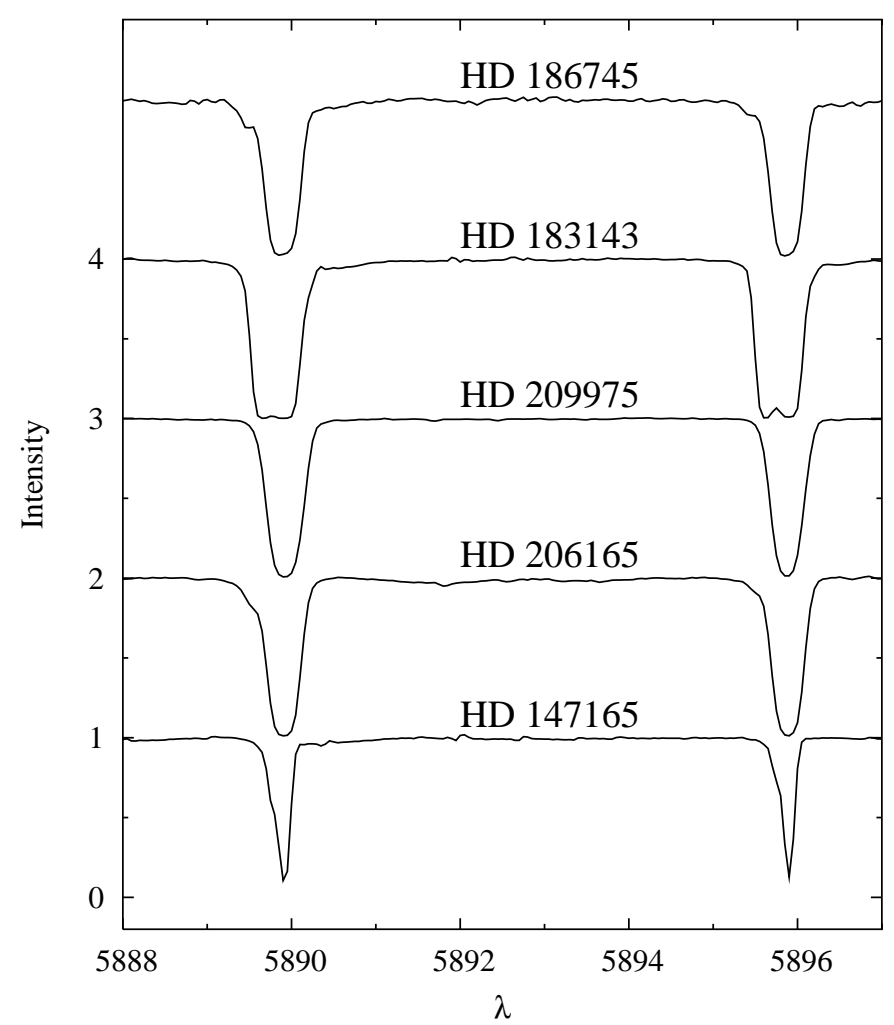

Fig. 4. Interstellar sodium lines in spectra of five stars. Note the Doppler splitting seen in the spectrum of HD 183143 and the widths of profiles in the case of higly reddened HD 183143 and 186745 . The widths of sodium lines in the spectra of the two stars HD 206165, 209975 from Cep OB2 association are comparable to those observed toward HD 186745. Note that sodium lines in the case of HD 147165 from Sco OB2 association are narrower than those in the spectra of four stars seen above.

those between weaker ones. The very good correlations between $\lambda \lambda 5809,6196,6614$ and 6660 may suggest that these DIBs possibly form the spectrum originating at the same molecule. But the value of the correlation coefficient between $\lambda \lambda 6660 / 5809$ shows that $\lambda 6660$ cannot be neglected. This DIB can belong to the spectrum of the same hypothetical molecule. In the case of $\lambda \lambda 6614 / 6397$ a rather poor correlation was obtained because the profile of the diffuse band $\lambda 6397$ is possibly blended with the stellar line in the spectra of target stars.

\subsection{The region $4460-8800 \AA$ in the spectra of $H D 183143$ and 186745}

The known spectrum of DIBs contains nearly 300 features in the spectral range 3800-8800 $\AA$ (Jenniskens \& Desért 1994; Galazutdinov et al. 2000). Most of them are found in the region 5500-8000 $\AA$. In the region outside of the observed limit of McDonald spectra (5600-7000 $\AA$ ), we can expect additional DIBs possibly connected with those that are well correlated. They can also fit the achieved sequences based on correlation analysis or form 


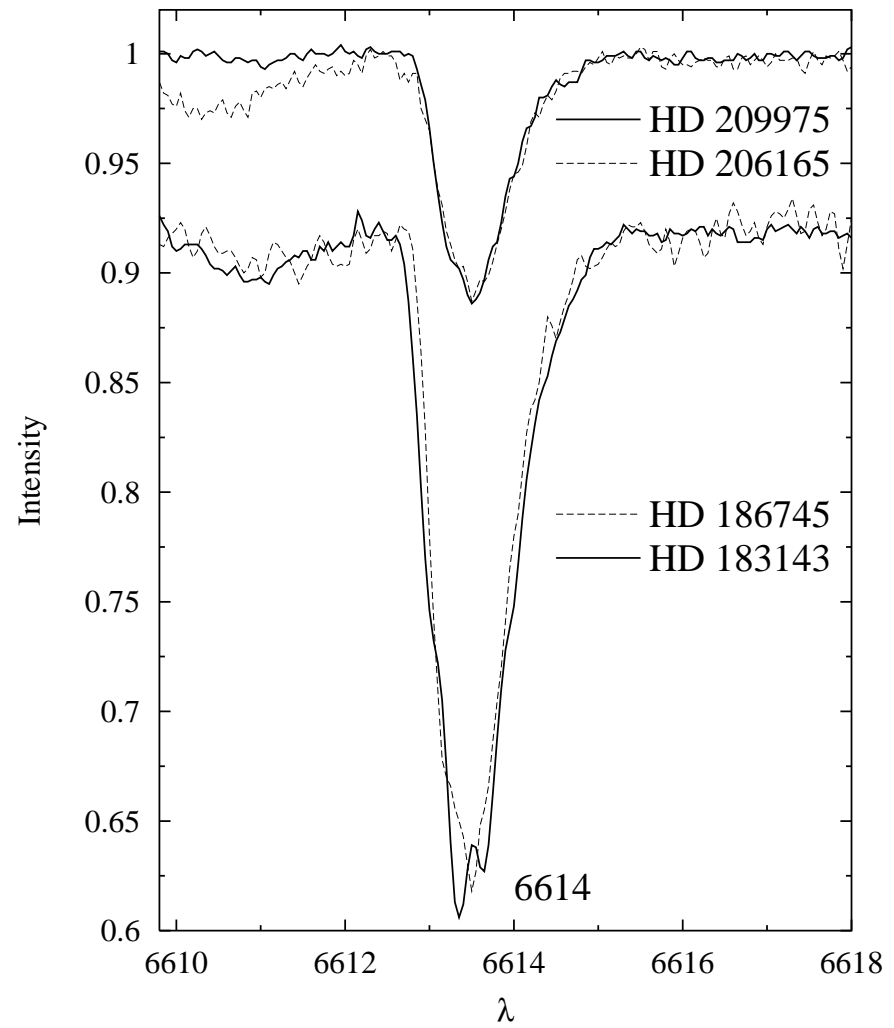

Fig. 5. The profiles of $\lambda 6614$ in spectra of four stars. The profile in the case of HD 183143 is Doppler-splitted. The central depths in the two sets of spectra are quite similar.

the different spectra. To test this, we analyzed the central depths of weak DIBs in the spectra of HD 183143 and HD 186745. Spectra of these stars were acquired in the range 4460-8800 $\AA$ with the aid of new coude echelle spectrometer at pic Terskol. We examined the regions 4460-5600 and 7000-8800 $\AA$ and found two features at 5404.5 and $7257.5 \AA$ whose central depth is nearly the same in the spectra of $\sigma$-type HD 183143 and $\zeta$-type HD 186745 (the $\lambda 5404.5$ is presented in Fig. 7). These diffuse bands can also form the spectral sequence with $\lambda \lambda 5809,6196,6614$ or 6660 . The association of the two features with the other ones is rather tentative, because of the small sample of objects. It is, however, interesting that no other similar suggestions can be made using the available material.

\section{Discussion}

\subsection{Positions of DIBs}

In the sample of 41 reddened stars, the light of which penetrates the $\sigma$ or $\zeta$ type interstellar clouds, the intensities of DIBs 5809, 6196, 6614 and 6660 are mutually very well correlated. Based on the values of correlation coefficients, seen in Table 3, we have rejected from the sample the following weak DIBs: $\lambda \lambda$ 5793, 5819, 5828 and 6397. A schematic spectrum of very well-correlated DIBs $\lambda \lambda$ 5809, 6196, 6614 and 6660, which was made by averaging the spectra of 41 reddened stars, is shown in

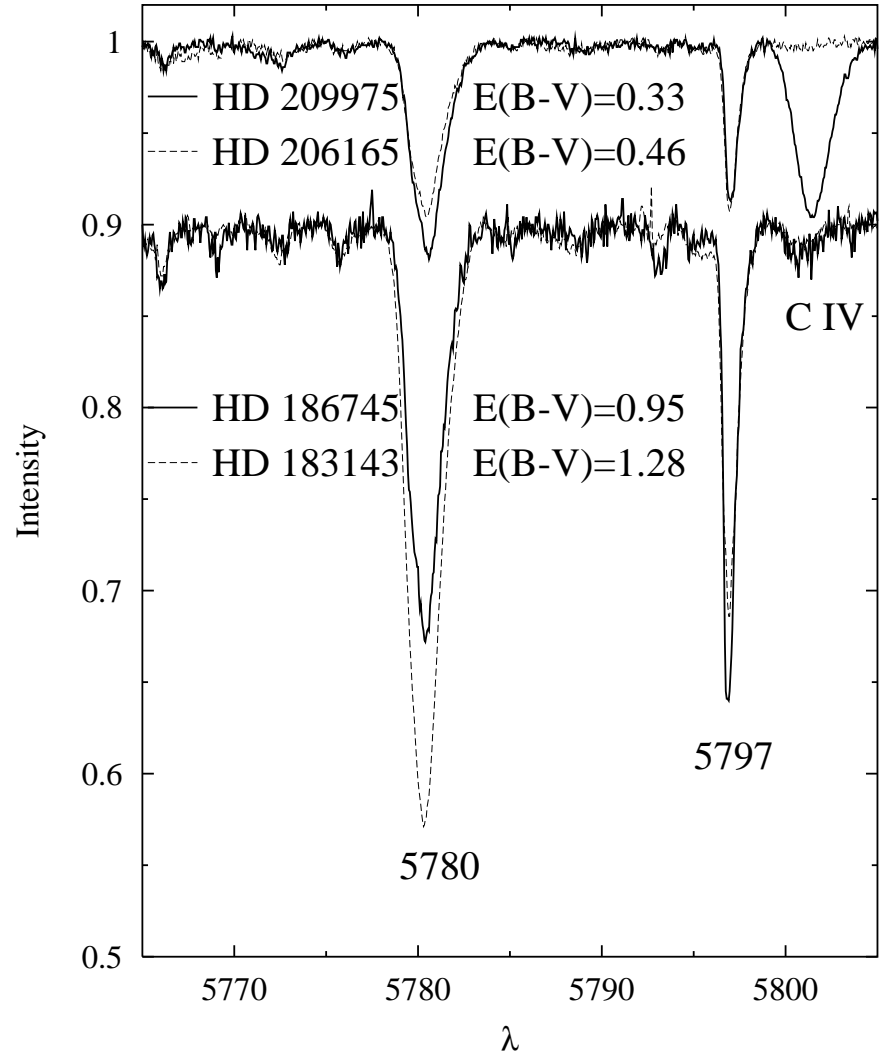

Fig. 6. The spectra of four stars in the region of $\lambda 5780$ and 5797. The 5780/5797 strength ratios in the cases of HD 183143 and 186745 are typical for $\sigma$ and $\zeta$-type clouds respectively. The stellar C IV line at $5801.313 \AA$ is clearly seen in the spectrum of HD 209975.

Fig. 8. Additionally, in Fig. 8 we present the positions of two DIBs at 5404.5 and $7257.5 \AA$ that have similar central depths in the spectra of two heavily reddened stars. The profile of each diffuse band lying in the wavelength range of the McDonald spectra (5600-7000 $\AA$ ) have been approximated with a Gaussian curve that has the same half width at full maximum. The constancy of the relative intensities of DIBs in the $\sigma$ as well as $\zeta$-type clouds suggests that they may originate from the same molecular carrier. If the DIBs 5809, 6196, 6614 and 6660 form the electronic spectrum of a molecule in the gas phase, the $\lambda 6660$ were the origin band of this spectrum. The remaining DIBs would correspond to the vibronic transitions of this hypothetical molecule. A question immediately appears whether we are able to identify this mysterious molecule, which would be responsible for the spectrum of DIBs 5809, 6196, 6614 and 6660 shown in Fig. 8? The most probable carriers of DIBs are radical species: linear (carbon chains), planar (PAH cations) and spherical (fullerene cations) based on a carbon skeleton. At present, the electronic spectra of several such molecular systems are known in the gas phase and many more such species have been studied in low temperature matrices (Fulara \& Krełowski 2000; Maier 1998; Salama et al. 1999); several tens of such molecular systems are known in the gas phase. Comparison of the electronic spectra of numerous radical species, built of a 
Table 3. Correlation coefficients with errors in brackets. Number of stars in each sample is shown at the bottom of each line.

\begin{tabular}{|c|c|c|c|c|c|c|c|c|}
\hline$\overline{\text { DIB }}$ & 6660 & 6614 & 6397 & 6196 & 5828 & 5819 & 5809 & 5793 \\
\hline 6614 & $\begin{array}{c}0.96(0.18) \\
36\end{array}$ & - & & & & & & \\
\hline 6397 & $\begin{array}{c}0.86(0.13) \\
30\end{array}$ & $\begin{array}{c}0.81(0.17) \\
33\end{array}$ & - & & & & & \\
\hline 6196 & $\begin{array}{c}0.92(0.14) \\
35\end{array}$ & $\begin{array}{c}0.96(0.17) \\
40\end{array}$ & $\begin{array}{c}0.73(0.11) \\
33\end{array}$ & - & & & & \\
\hline 5828 & $\begin{array}{c}0.66(0.31) \\
26\end{array}$ & $\begin{array}{c}0.59(0.32) \\
28\end{array}$ & $\begin{array}{c}0.79(0.30) \\
22\end{array}$ & $\begin{array}{c}0.60(0.31) \\
27\end{array}$ & - & & & \\
\hline 5819 & $\begin{array}{c}0.58(0.25) \\
25\end{array}$ & $\begin{array}{c}0.48(0.27) \\
29\end{array}$ & $\begin{array}{c}0.79(0.24) \\
26\end{array}$ & $\begin{array}{c}0.49(0.25) \\
28\end{array}$ & $\begin{array}{c}0.82(0.37) \\
23\end{array}$ & - & & \\
\hline 5809 & $\begin{array}{c}0.96(0.10) \\
24\end{array}$ & $\begin{array}{c}0.95(0.15) \\
26\end{array}$ & $\begin{array}{c}0.81(0.08) \\
23\end{array}$ & $\begin{array}{c}0.90(0.11) \\
35\end{array}$ & $\begin{array}{c}0.73(0.29) \\
18\end{array}$ & $\begin{array}{c}0.56(0.23) \\
21\end{array}$ & - & \\
\hline 5793 & $\begin{array}{c}0.77(0.12) \\
29\end{array}$ & $\begin{array}{c}0.75(0.16) \\
32\end{array}$ & $\begin{array}{c}0.79(0.10) \\
29\end{array}$ & $\begin{array}{c}0.78(0.11) \\
31\end{array}$ & $\begin{array}{c}0.82(0.29) \\
22\end{array}$ & $\begin{array}{c}0.73(0.24) \\
24\end{array}$ & $\begin{array}{c}0.77(0.06) \\
23\end{array}$ & - \\
\hline
\end{tabular}



Fig. 7. Diffuse band at $5404.5 \AA$ in the spectra of HD 183143 and 186745 . The central depth of this DIB in both spectra is nearly the same.

carbon skeleton, with the spectrum of DIBs presented in Fig. 8, shows a lack of any correspondence.

Let us consider now whether the spectrum of DIBs (Fig. 8), that preserves nearly constant intensity ratios among the sample of 41 reddened stars, can originate from one molecule? If the $\lambda 6660$ is the origin band of a hypothetical molecule, the first vibronic level of this molecule should be located $104 \mathrm{~cm}^{-1}$ above the origin (the $\lambda 6614$ ). In the electronic spectra of chemical species that are considered as the carriers of DIBs, the bending modes of linear molecules and the torsional modes of planar systems (e.g. PAH cations) possess the lowest vibronic energies. However, these energies exceed by a factor of two the energy of the first vibronic band $(\lambda 6614)$ in the synthetic spectrum of DIBs shown in Fig. 8 (Freivogel et al. 1995; Salama \& Allamandola 1995; Szczepański et al. 1993). Another peculiarity which is apparent in the spectrum of DIBs (Fig. 8) is the relative intensity of the first vibronic band $(\lambda 6614)$ with respect to the band $(\lambda 6660)$ that exceeds by a factor of three the intensity of the origin band. In a case of such a strong vibronic band, an additional DIB ought to be expected in the $6570 \pm 10 \AA$ spectral range,

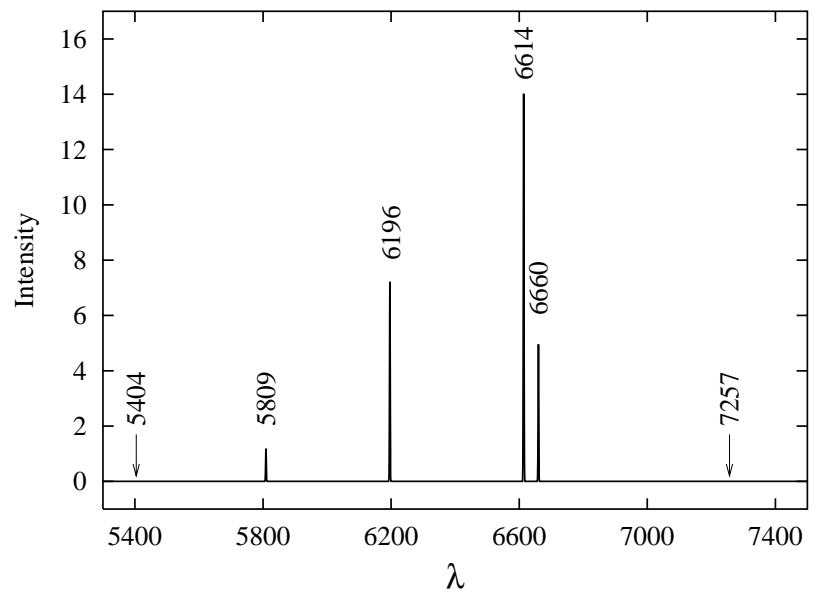

Fig. 8. The schematic spectrum of $\lambda \lambda 5809,6196,6614$ and 6660 achieved after averaging of the central depths of these features in the spectra of 41 stars seen in Table 1. The shape of each diffuse band was approximated with a Gaussian curve of the same half width at full maximum. Additionally we present the positions of two DIBs at 5404.5 and $7257.5 \AA$.

which would be separated by ca. $200 \mathrm{~cm}^{-1}\left(2 \times 104 \mathrm{~cm}^{-1}\right)$ from the origin band and it would be at least as intense as the $\lambda 6397$. A lack of such a band (possibly lying in the vicinity of a strong $\mathrm{H}_{\alpha}$ line) in the spectra of 41 reddened stars, the intensity of which correlates with the intensities of the $\lambda \lambda 6614$ and 6660 , seems to suggest that the $\lambda \lambda 6614$ and 6660 originate from different molecules.

Let us consider another hypothesis, assuming that $\lambda 6614$ would be the origin band of an other molecule. The intensity of the weak $\lambda 6397$ band correlates to a similar extent with $\lambda \lambda 6614$ and 6660 . The other possibility is that the bands $\lambda \lambda 6614$ and 6660 are the origins of two electronic transitions of the same molecule. Hovewer, this is an unusual case because the electronic states would be very close each other. We have to consider the correlation of the strong $\lambda 6196$ with $\lambda \lambda 6614$ and 6660 . As we can see in Table 3 the central depth of $\lambda 6196$ correlates very well with the central depth of $\lambda \lambda 6614$ and 6660 within the sample of 41 reddened stars. The correlation analysis 


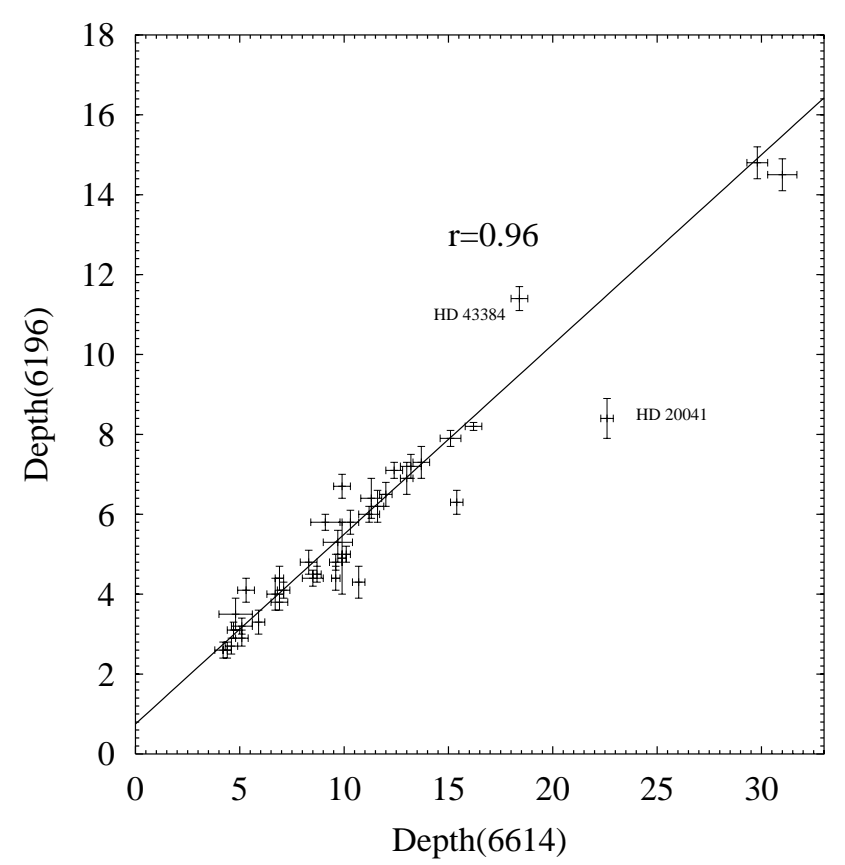

Fig. 9. The correlation plot between $\lambda \lambda 6196$ and 6614 . Additional information on the two datapoints that miss the correlation can be found in Motou et al. (1999).

suggests that $\lambda 6614$ or $\lambda 6660$ can be the origin band of the same molecule. The first case, $\lambda \lambda 6614 / 6196$ is very striking, with the correlation coefficient $r=0.96$ (Fig. 9).

If $\lambda 6196$ forms the electronic spectrum of a molecule with $\lambda 6614$ or $\lambda 6660$, the $\lambda 6196$ should be the vibronic band of energy 1020 or $1124 \mathrm{~cm}^{-1}$ respectively. The intensity of $\lambda 6196$ is considerable with respect to the $\lambda 6614$ or $\lambda 6660$. Therefore, we can predict the overtone band in the $5820 \pm 10 \AA$ or the $5793 \pm 10 \AA$ spectral range that would be spaced from the origin towards the short-wavelength region by $2 \times 1020$ or $2 \times 1124 \mathrm{~cm}^{-1}$ respectively. In the spectral region indicated above, we have found the interstellar features at $\lambda \lambda$ 5793, 5809, 5819 and 5828 but only $\lambda 5809$ is very well correlated with $\lambda \lambda 6196$ and 6614 . In the case of $\lambda 6660$ the correlation is also very good. This is a very exciting result which shows that not only the intensities of $\lambda \lambda 6614,6196,5809$ are very well correlated but also that these bands are spaced from each other by ca. $1000 \mathrm{~cm}^{-1}$. These bands can be assigned as the origin, the vibronic and the overtone band of a molecule respectively (Hollas 1998). However, we are not able to deduce from this spectrum what kind of molecule can be responsible for these three DIBs, since the frequency of $1000 \mathrm{~cm}^{-1}$ is not very characteristic for the fundamental modes of molecules (Silverstein et al. 1974). Due to worse correlation of the $5793 \AA$ band with the $\lambda \lambda 6196$ and 6660 we can exclude the possibility that these three bands originate from the same carrier.

In order to prove whether other DIBs form the spectrum of this hypothetical molecule, the search for such DIBs should be extended up to the $5300 \AA$ wavelength that lies about $3700 \mathrm{~cm}^{-1}$ above the origin band $(\lambda 6614)$. The highest vibrational frequency of energy at about

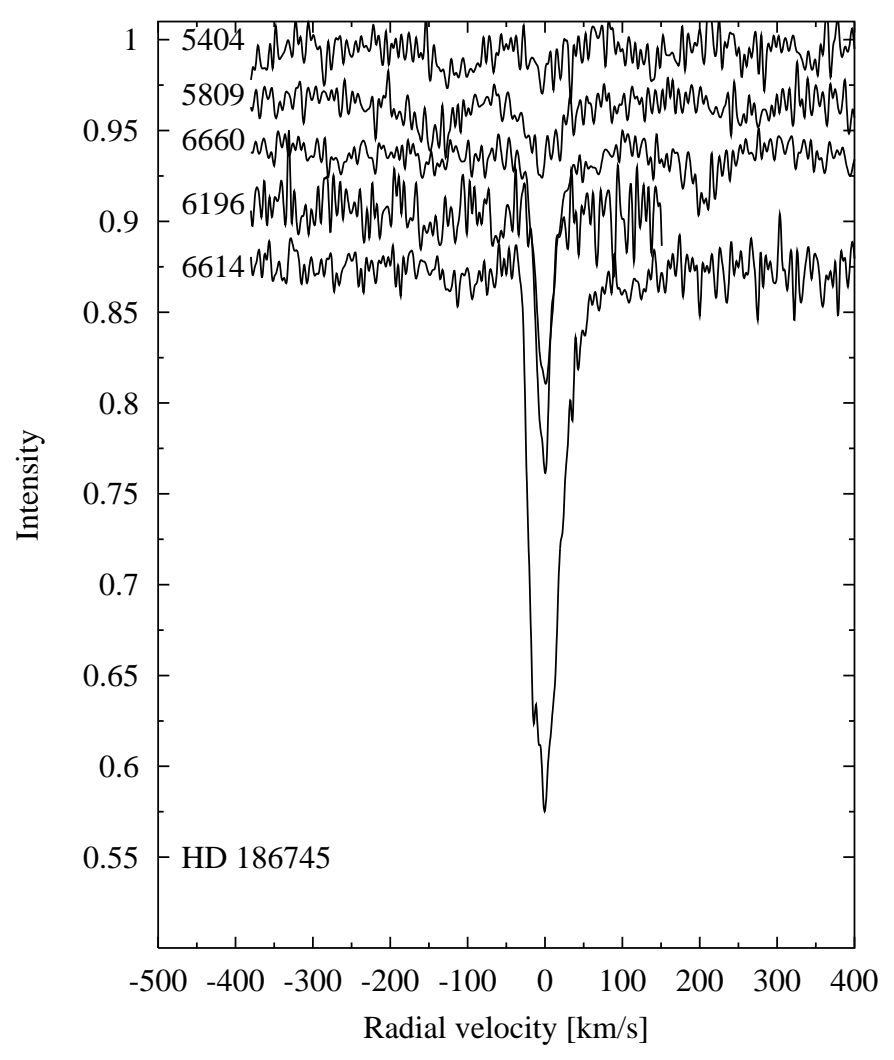

Fig. 10. The profiles of $\lambda \lambda 5404.5,5809,6196,6614$ and 6660 in the spectrum of highly-reddened HD 186745. In these cases we do not see any Doppler-splitting and the difference in FWHM in the case of $\lambda \lambda 6196$ and 6614 is easily seen.

$3700 \mathrm{~cm}^{-1}$ observed in any molecular system (except of $\mathrm{H}_{2}$ ) corresponds to the excitation of the $\mathrm{OH}$ stretching mode of a molecule (Silverstein et al. 1974). In the short-wavelength spectral range $(4400-5600 \AA)$, we have found the $\lambda 5404.5$ band (shown in Fig. 7) that has a similar intensity in the spectra of the HD 183143 and HD 186745 stars. Unfortunately, we were not able to perform the correlation studies with the other selected DIBs due to the very small number of target stars (two). The vibrational frequency of this band with respect to the origin band $(\lambda 6614)$ is equal to $3385 \mathrm{~cm}^{-1}$. This value is too high for the $\mathrm{C}-\mathrm{H}$ stretching mode and is too low for the $\mathrm{OH}$ stretching mode. Concluding, this band probably does not originate from the same carrier as the $\lambda \lambda 6614,6196$, $5809 \AA$ do.

To show if $\lambda 6614$ is the origin band we have to examine the long-wavelength spectral range up to $8800 \AA$ (about $3700 \mathrm{~cm}^{-1}$ below the $6614 \AA$ band). In the near infrared spectral region we have found only one very weak band at $7257.5 \AA$, which has a similar intensity in the spectra of the HD 183143 and HD 186745 stars, but the correlations with the other selected DIBs have not been carried out for the same reason as for the $5404.5 \AA$ band. This band is spaced by 1236,1340 and $2361 \mathrm{~cm}^{-1}$ from the 6660,6614 and $6196 \AA$ bands respectively. Such energy intervals are not present in the spectrum of the selected DIBs. The low intensity of this band with respect to the 
$6614 \AA$ band gives a further argument against a common origin for this band with the $6614,6196,5809 \AA$ band set.

\subsection{Profiles of DIBs}

The contour shape of an electronic band with unresolved rotational structure can be specific to a given molecule. Intuitively, it seems obvious that the contours of the vibronic bands that form the electronic spectrum of a molecule should be similar. The profile of $\lambda 6614$ shows internal substructures and is asymmetric with a characteristic steep ascendant slope on the short-wavelength edge and slowly descendant portion on the long-wavelength edge. The profile of $\lambda 6196$ is sharp and almost symmetrical. It does not show any pattern substructures. These differences were fully described by Krełowski \& Schmidt (1997).

Figure 10 shows that the full width at half maximum (FWHM) of $\lambda 6196$ is about two times smaller than the $F W H M$ of $\lambda 6614$. The question arises whether such a large difference in the FWHM of the bands in the case when they originate at the same carrier is possible. The shape of rotationally unresolved bands depends on several factors, such as temperature, rotational constants $B^{\prime}$ and $B$ " in the ground and the excited electronic states etc. For a given electronic transition, possible differences in the shape of vibronic bands are dominated by the differences in the rotational constants $B^{\prime}$ in the vibronic states of a molecule.

The simulations of the rotational contours of the electronic absorption bands of a hypothetical molecule have been performed in order to visualise the differences in the profiles of the vibronic bands. The profiles of the vibronic bands of a molecule can differ in their shape due to small changes in geometry in the vibronic states of this molecule. The simulations have been carried out for the simplest model: the $\Sigma-\Sigma$ electronic transition of a linear gas-phase molecule built of 14 heavy atoms $(\mathrm{C}, \mathrm{N}, \mathrm{O})$, at the temperature of $60 \mathrm{~K}$. Four cases have been considered where the rotational constant $B^{\prime}$ in the vibronic state is equal to $B$ " or is smaller by $0.5,1,2 \%$ with respect to the rotational constant $B$ " in the ground state. The results of simulations are shown in Fig. 11. The integral intensity of the vibronic bands was the same in all cases. As can be seen in Fig. 11 even small change of the rotational constant $B$ ' leads to large change of the rotational contour of the band. Small changes in geometry of a molecule induce large changes in the widths as well as in the intensities of the substructures that form the profile of the vibronic band. This is very well illustrated in Fig. 11, where decreasing the rotational constant $B^{\prime}$ by $0.5 \%$ causes reduction by a factor of 2 in the half-width of the band head (the strong substructure seen on the short-wavelength edge of the profile) and considerable increase in intensity of this substructure.

The simulations of the rotational contours of a linear molecule show that DIBs which differ by a factor of up to two in their half-widths e.g. $\lambda \lambda 6196$ and 6614 bands, can originate in the same carrier.

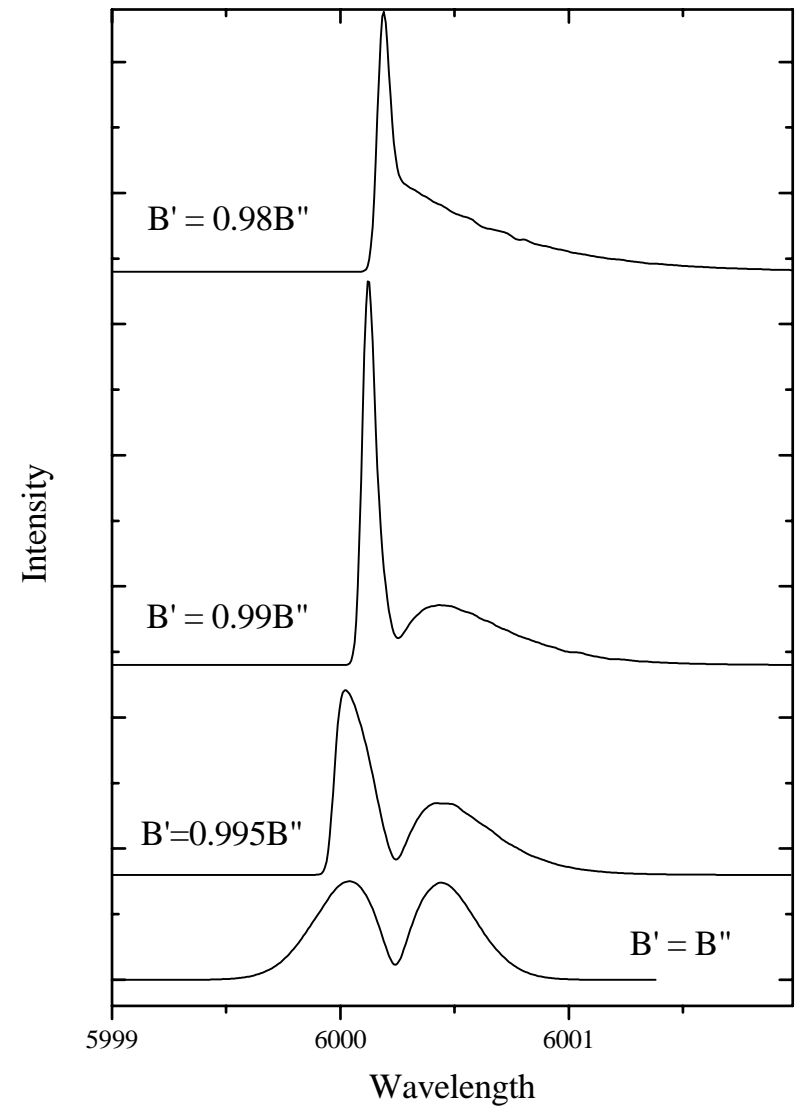

Fig. 11. The simulations of rotational contours of the rotational absorption bands of a hypotetical linear gas-phase molecule. This model was achieved for the $\Sigma-\Sigma$ electronic transition of a molecule built of 14 heavy atoms $(\mathrm{C}, \mathrm{N}, \mathrm{O})$ at a temperature of $60 \mathrm{~K}$. In the individual cases the rotational constant $B$ ' in the vibronic state is equal to $B$ " or is smaller by $0.5,1,2 \%$ (from the bottom) with respect to the rotational constant $B$ " in the ground state. The integral intensity of the vibronic bands was the same in all cases.

\section{Conclusions}

A search for sets of DIBs possibly originating in the same carrier is very difficult because spectra of earlytype stars suffer many contaminations. Nevertheless, correlation analysis is a useful tool to retrieve sets of features possibly forming spectra of single interstellar species. It is, however, not sufficient, and additional analyzes are necessary to make sure that a suggested set of DIBs originated in fact in a single molecular species.

The above considerations led us to infer the following conclusions:

1. The correlation analysis based on the sample of 41 stars in the region $5600-7000 \AA$ and the analysis of DIB positions showed that $\lambda \lambda 6614$ (6660) may be the origin band, and $\lambda \lambda 6196,5809$ two vibronic bands of the spectrum of the same molecule.

2. The simulations of a hypothetical gas-phase molecule showed that diffuse bands which differ by a factor of 
two in their half-widths can still originate in the same carrier.

Apparently, the very rich spectrum of interstellar clouds, consisting of numerous unidentified features - the DIBs is composed of spectra of single species, containing typically strong and weak features. A more complete analysis of mutual relations between all detectable DIBs is highly desirable. It requires much larger samples of observed targets as well as high resolution and high $S / N$ spectra; such material is very hard to get. Any analysis of the DIB spectrum also requires a very careful consideration of all possible contaminations of the measured features.

Acknowledgements. This paper was supported by the Polish State Committee for Scientific Research under grants 2.P03D. 011.15 and 2.P03D.008.16. We are very grateful to G. A. Galazutdinov and P. A. Musaev who provided us with the spectra of HD 183143 and 186745. We also thank the referee for valuable comments.

\section{References}

Cami, J., Sonnentrucker, P., Ehrenfreund, P., \& Foing, B. H. 1997, A\&A, 326, 822

Chlewicki, G., de Groot, M. S., van der Zwet, G. P., et al. 1987, A\&A, 173, 131

Ehrenfreund, P., Foing, B. H., d'Hendecourt, L., Jenniskens., P., \& Désert, F. X. 1995, A\&A, 229, 213

Foing, B. H., \& Ehrenfreund, P. 1994, Nature, 369, 296

Foing, B. H., \& Ehrenfreund, P. 1997, A\&A, 317, L59

Freivogel, P., Fulara, J., Jakobi, M., Forney, D., \& Maier, J. P. 1995, J. Chem. Phys., 103, 54

Fulara, J., Lessen, D., Freivogel, P., \& Maier, J. P. 1993, Nature, 439, 366

Fulara, J., \& Krełowski, J. 2000, New Astron. Rev., 44, 581

Galazutdinov, G. A., Preprint Spets. Astrof. Obs., No. 92
Galazutdinov, G. A., Krełowski, J., Musaev, F. A., Ehrenfreund, P., \& Foing, B. H. 2000, MNRAS, 317, 750

Galazutdinov, G. A., Musaev, F. A., Krełowski, J., \& Walker, G. A. H. 2000, PASP, 112, 648

Heger, M. L. 1922, Lick Obs. Bull., 10, 146

Herbig, G. H. 1975, ApJ, 196, 129

Hollas, J. M. 1998, High Resolution Spectroscopy (J. Wiley \& Sons)

Herbig, G. H., \& Soderblom, D. R. 1982, ApJ, 252, 610

Jenniskens, P., \& Désert, F.-X. 1994, A\&AS, 106, 39

Krełowski, J., \& Walker, G. A. H. 1987, ApJ, 312, 860

Krełowski, J., \& Sneden, C. 1993, PASP, 105, 1141

Krełowski, J., Sneden, C., \& Hiltgen, D. 1995, Planet. Space Sci., 43, 1195

Krełowski, J., \& Schmidt, M. 1997, ApJ, 477, 209

Krełowski, J., Schmidt, M., \& Snow, T. P. 1997, PASP, 109, 1135

Maier, J. P. 1998, J. Chem. Phys., 102, 3462

McCarthy, J. K., Sandiford, B. A., Boyd, D., \& Booth, J. 1993, PASP, 105, 881

Motou, C., Krełowski, J., d'Hendecourt, L., \& Jamroszczak, J. 1999, A\&A, 351, 680

Motylewski, T., Linnartz, H., Vaizert, O., et al. 2000, ApJ, 531, 312

Musaev, F. A. 1993, Piśma V. Astron. Zh., 19, 776

Papaj, J., Krełowski, J., \& Wegner, W. 1993, A\&A, 273, 575

Salama, F., Galazutdinov, G. A., Krełowski, J., Allamandola, L. J., \& Musaev, F. A. 1999, ApJ, 526, 265

Salama, F., \& Allamandola, L. J. 1991, J. Chem. Phys., 94, 6964

Silverstein, R. M., Bassler, G. C., \& Morril, T. C. 1974, Spectroscopic Identification of Organic Compounds (J. Wiley \& Sons)

Szczepański, J., Vala, M., Talbi, D., Parisel, O., \& Elinger, Y. 1993, J. Chem. Phys., 98, 4494

Tuairisg, S.Ó., Cami, J., Foing, B. H., Sonnentrucker, P., \& Ehrenfreund, P. 2000, A\&AS, 142, 225

Weselak, T., Schmidt, M., \& Krełowski, J. 2000, A\&AS, 142, 239

Westerlund, B. E., \& Krełowski, J. 1988, A\&A, 203, 134 